\title{
Every Nook and Cranny
}

\author{
Siddharth Jain"; James Pile2; Nitin Gupta³; Nathan Houchens ${ }^{4,5}$; Rama Chaudhry6; Neeraj Nischal1*
}

This icon represents the patient's case. Each paragraph that follows represents the discussant's thoughts.

'Department of Medicine, All India Institute of Medical Sciences, New Delhi, India; ${ }^{2}$ Department of Medicine, MetroHealth Medical Center at Case Western Reserve University, Cleveland, Ohio; ${ }^{3}$ Departments of Medicine and Microbiology, All India Institute of Medical Sciences, New Delhi, India; ${ }^{4}$ Medicine Service, Veterans Affairs Ann Arbor Healthcare System, Ann Arbor, Michigan; ${ }^{5}$ Department of Internal Medicine, University of Michigan Medical School, Ann Arbor, Michigan; 'Department of Microbiology, All India Institute of Medical Sciences, New Delhi, India.

A 46-year-old man presented to the emergency room in the postmonsoon month of September with a seven-day history of high fevers as well as a four-day history of a dry cough, dyspnea, and progressive rash. The patient reported no chest pain, hemoptysis, chest tightness, palpitations, wheezing, orthopnea, paroxysmal nocturnal dyspnea, or leg swelling. He lived and sought healthcare in Delhi, India.

Fever followed by a progressive but as yet uncharacterized rash and pulmonary symptoms in a middle-aged man suggests a host of possibilities. While it is tempting to ascribe his symptoms to an infectious process, especially a "tropical" infection based on his residence in Delhi, the location may simply represent a red herring. Potential infections can be divided into those endemic to the Indian subcontinent, and those encountered more globally. The former include diseases such as measles and dengue, while the latter include entities such as Mycoplasma pneumonia, varicella, and acute human immunodeficiency virus (HIV) infection. Noninfectious categories of diseases that should be considered include drug reactions and rheumatologic processes. Several rheumatologic diseases, including granulomatosis with polyangiitis, eosinophilic granulomatosis with polyangiitis, and systemic lupus erythematosus (SLE) may present with fever, rash, and pulmonary symptomatology.

A history of the patient's exposures, both environmental and pharmaceutical, should be obtained. More information regarding his immunization history, rash characteristics (distribution and nature of the lesions), and other salient exam findings such as organomegaly and joint abnormalities will be helpful.

Fever reached a maximum of $103^{\circ}$ Fahrenheit and was associated with chills but not rigors. There were several fever spikes daily, relieved completely with antipyretics. The patient's dyspnea was predominantly noted on exertion, nonpleuritic, not temporally related to cough, and progressively

*Corresponding Author: Neeraj Nischal; E-mail: neerajnischal@gmail.com; Telephone: +919811484060

Published online first March 20, 2019.

Received: September 4, 2018; Revised: December 22, 2018;

Accepted: January 6, 2019

๑ 2019 Society of Hospital Medicine DOI 10.12788/jhm.3163 worsening over three days. The skin lesions were first noticed on his trunk and were described as reddish, flat, and pinpoint size. However, the rash spread to the face and extremities sparing the palms and soles. There was no bleeding, nausea, vomiting, abdominal pain, change in bowel habits, dysuria, headache, photophobia, neck stiffness, or joint pain.

The patient reported no significant past medical history, took no medications, and had no recent travel outside of Delhi, India in the past year. He was married and monogamous. He had no pets nor did he report any contact with animals. He did not use tobacco, alcohol, or illicit substances. He did not remember being bitten by an insect. He worked as a software engineer. There was no history of similar illness in the patient's family or at his workplace. He had no history of recent blood transfusion or immunization (including MMR and Tdap).

Several noninfectious and inflammatory conditions can explain his symptoms. Eosinophilic granulomatosis with polyangiitis is considerably less likely in the absence of asthma, and vasculitic processes, in general, are less likely given the nongravity dependent nature of the rash. SLE and sarcoidosis are possible causes of a systemic inflammatory illness presenting acutely with fever, rash, and pulmonary symptoms.

The patient's expanded history makes several infections less likely. Although much of the presentation is consistent with measles, the initial appearance of the truncal rash is atypical, and there is no mention of coryza or conjunctivitis. Likewise, the description of the exanthem is not suggestive of varicella, and dengue and chikungunya are much less likely in the absence of a headache and arthralgias. Other infections including leptospirosis and scrub typhus are possible, and both might be contracted in greater Delhi. Typhoid is another infectious syndrome endemic to the Indian subcontinent that should be considered. The presence of rash involving the face and extremities would be highly atypical, however; and the presence of dyspnea and the absence of a headache argue against typhoid. Acute HIV infection and Mycoplasma pneumonia remain possible diagnoses. Toxic shock syndrome is possible, but a faster and fulminant course would be expected.

On physical examination, the temperature was $103^{\circ}$ Fahrenheit, heart rate was 120 beats per minute and 
regular, respiratory rate was 24 breaths per minute, blood pressure was $100 / 60 \mathrm{~mm} \mathrm{Hg}$, and resting oxygen saturation was $93 \%$ while breathing ambient air. He appeared uncomfortable. Jugular venous pulse was elevated at $10 \mathrm{~cm} \mathrm{H}_{2} \mathrm{O}$. Mild icterus was present, but there was neither conjunctival congestion nor subconjunctival hemorrhage. S1 and S2 heart sounds were loud, but there were no murmurs. Chest auscultation revealed bilateral basal coarse crackles. The abdominal right upper quadrant was mildly tender to palpation, and the liver edge was palpable $2 \mathrm{~cm}$ below the subcostal margin. There was neither splenomegaly nor peripheral lymphadenopathy. Kernig and Brudzinski signs were negative, and there were no focal neurological deficits. A generalized, nonpalpable, maculopapular and petechial rash was present on the face, extremities, and trunk.

The patient's presentation must now incorporate the additional findings of bibasilar chest crackles, maculopapular/petechial rash, icterus, modest hypoxia, and hepatomegaly. Some of the noninfectious entities already mentioned (SLE and sarcoidosis) remain possible explanations. Hemophagocytic lymphohistiocytosis $(\mathrm{HLH})$ may also explain most of the patient's presenting signs and symptoms, and several other infectious diseases account for his presentation. Scrub typhus (or a more uncommon rickettsia disease, Indian tick typhus), leptospirosis, and perhaps infective endocarditis seem most likely to provide a unifying diagnosis for the symptoms mentioned above. Leptospirosis presents in a minority of instances as a severe illness known as Weil disease, characterized by several of this patient's findings including icterus, kidney injury, and pulmonary symptoms. However, the rash is relatively uncommon in leptospirosis and when present, is usually more localized. The patient's rash as described is not typically expected in infective endocarditis, although high-grade Staphylococcus aureus bacteremia will occasionally present with a diffuse rash that may be confused with that of meningococcemia. The etiology of the patient's elevated jugular venous pressure is not readily apparent, with the cardiac examination making acute valvular insufficiency much less likely. Myocarditis, however is possible in the setting of several of the diseases listed above, including leptospirosis, scrub typhus, SLE, and dengue.

In addition to basic laboratory studies and a chest radiograph, multiple sets of blood cultures should be obtained, along with a transthoracic echocardiogram and a ferritin level. The evidence to support leptospirosis and scrub typhus is strong enough to justify empiric use of doxycycline once the blood cultures are obtained, especially given the difficulty in definitively diagnosing these diseases in a timely fashion.

Laboratory analysis revealed a total leukocyte count of $13,600 / \mathrm{uL}$ (85\% neutrophils), hemoglobin $10 \mathrm{~g} / \mathrm{dL}$, and platelet count 35,000/uL. Absolute eosinophil count was 136/ uL. Serum chemistry showed sodium of $145 \mathrm{meq} / \mathrm{L}$, potassium $4.1 \mathrm{meq} / \mathrm{L}$, blood urea nitrogen $80 \mathrm{mg} / \mathrm{dL}$, creatinine 1.6 $\mathrm{mg} / \mathrm{dL}$, aspartate transaminase (AST) $44 \mathrm{U} / \mathrm{L}$ (normal, 0-40), alanine transaminase (ALT) $81 \mathrm{U} / \mathrm{L}$ (normal, 0-40), direct bilirubin $3 \mathrm{mg} / \mathrm{dL}$, and indirect bilirubin $3 \mathrm{mg} / \mathrm{dL}$. Lactate dehydro- genase, alkaline phosphatase, albumin, and coagulation studies were normal. Erythrocyte sedimentation rate (ESR) was 42 $\mathrm{mm}$ (normal, 0-25) and highly sensitive $\mathrm{C}$-reactive protein was $42 \mathrm{mg} / \mathrm{L}$ (normal, 0-10). Arterial blood gas on ambient air revealed a $\mathrm{pH}$ of 7.52, $\mathrm{PaCO}_{2} 24 \mathrm{~mm} \mathrm{Hg}, \mathrm{PaO}_{2} 55 \mathrm{~mm} \mathrm{Hg}$, and bicarbonate $20 \mathrm{meq} / \mathrm{L}$. Urinalysis was normal. Blood cultures were obtained. Electrocardiogram (ECG) showed regular narrow complex tachycardia with incomplete left bundle branch block. Old ECGs were not available for comparison. Chest radiograph showed bilateral air space opacities with evidence of vein cephalization. Abdominal and pelvis ultrasonography showed pericholecystic fluid and mild hepatomegaly, but no free fluid, pleural effusion, or evidence of cholecystitis. Point of care immunochromatographic rapid malarial antigen detection test (detects Plasmodium falciparum, Plasmodium vivax, Plasmodium malaria, and Plasmodium ovale) was negative.

Most of the findings described are commonly observed in both scrub typhus and leptospirosis, including cytopenias, parenchymal infiltrates, hepatomegaly, elevated transaminases and bilirubin, cardiac involvement, fever, and rash. The rash described is more consistent with scrub typhus than with leptospirosis. The absence of a headache and joint findings argue modestly against these diagnoses. Likewise, HLH provides an adequate explanation for most of the patient's symptoms, signs, and test results. These include fever, lung involvement, rash, hepatomegaly, elevated bilirubin, and cytopenias; however, leukocytosis and cardiac involvement are less characteristic. SLE also provides a satisfactory explanation for much of the symptoms, although the rash characteristics, normal urinalysis, and leukocytosis make this diagnosis less likely.

Additional testing that should be performed includes serum antinuclear antibody (ANA) and ferritin, since the latter may be markedly elevated in the setting of $\mathrm{HLH}$. Bone marrow aspirate and biopsy should be performed looking specifically for evidence of hemophagocytosis. Finally, a transthoracic echocardiogram (TTE) should be performed to assess evidence of myocardial dysfunction as it may alter the therapeutic approach, although the results will be unlikely to differentiate between the preceding considerations.

Troponin I was negative, but N-terminal probrain natriuretic peptide was elevated at $20,000 \mathrm{pg} / \mathrm{mL}$ (normal, 0-900). D-dimer was negative. TTE showed left ventricular ejection fraction (LVEF) of $35 \%$ with global left ventricular hypokinesis. On three separate examinations, the peripheral blood smear did not show malarial parasites, atypical lymphocytes, or schistocytes. Three sets of blood cultures, testing for bacteria and fungi, were sterile. A throat culture was sterile. Widal test, as well as Leptospira and Mycoplasma serologies, were negative. Serology for Legionella pneumophila was positive, but the urinary antigen testing was negative. Antibodies to HIV 1 and 2 and anti-hepatitis $C$ virus (HCV) antibody were negative. Dengue IgM ELISA (qualitative) returned positive. 

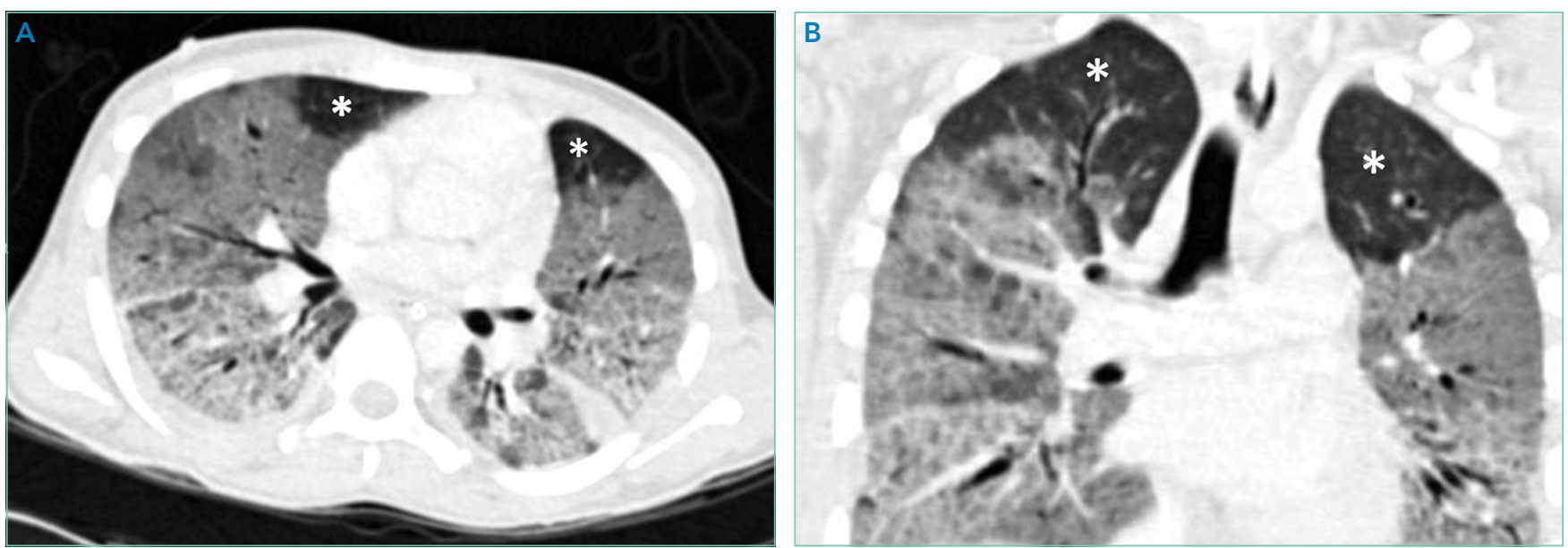

FIG 1. Axial (A) and coronal (B) computed tomography of the thorax showing extensive ground-glass opacities and consolidation sparing the nondependent portions of the lungs (apices and anterior segments, asterisks).

Despite the absence of arthralgias, myalgias, headache, and retro-orbital pain, a positive dengue IgM ELISA supports acute dengue infection, provided the patient did not experience an unexplained febrile illness in the previous months. Most of his presentation may be explained by dengue, including fever, rash, liver abnormalities, myocardial dysfunction, and thrombocytopenia. The bilateral airspace opacities seen on chest radiograph also fit reasonably provided these actually reflect pulmonary edema. Leukocytosis (as opposed to leukopenia) is highly unexpected in dengue, but its presence could be an outlier.

If dengue does indeed explain the entire presentation, defervescence should have occurred by the time the blood cultures and serologic studies returned. Also, by that time, the patient would be expected to demonstrate evidence of improvement, barring the appearance of the serious complications of dengue hemorrhagic fever/dengue shock syndrome. Should fever persist and signs of recovery fail to materialize, the possibility of a superimposed process will need to be considered. Of note, the sensitivity of Leptospira serology early in the course of illness is low, and leptospirosis is thus not yet excluded.

$\stackrel{\infty}{\infty}$

A presumptive diagnosis of severe dengue fever was made, based on evidence of pulmonary edema and sepsis. The patient was managed conservatively with oral fluid restriction, low dose of diuretics, and supplemental oxygenation. The patient was also given levofloxacin for possible legionellosis. Despite these therapies, the patient had no improvement in 24 hours. His tachypnea increased, and his measured $\mathrm{PaO}_{2}$ to $\mathrm{FIO}_{2}(\mathrm{P}: \mathrm{F})$ ratio decreased to 230 from 285 on admission. This prompted the initiation of BiPAP at $10 \mathrm{~cm}$

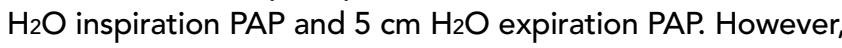
he did not tolerate BiPAP, and his P:F ratio decreased to below 200.

The patient was transferred to the intensive care unit and underwent elective intubation with mechanical ventilation. Axial and coronal computed tomography of the thorax (Figure $1 \mathrm{~A}$ and $1 \mathrm{~B}$, respectively) showed extensive ground-glass

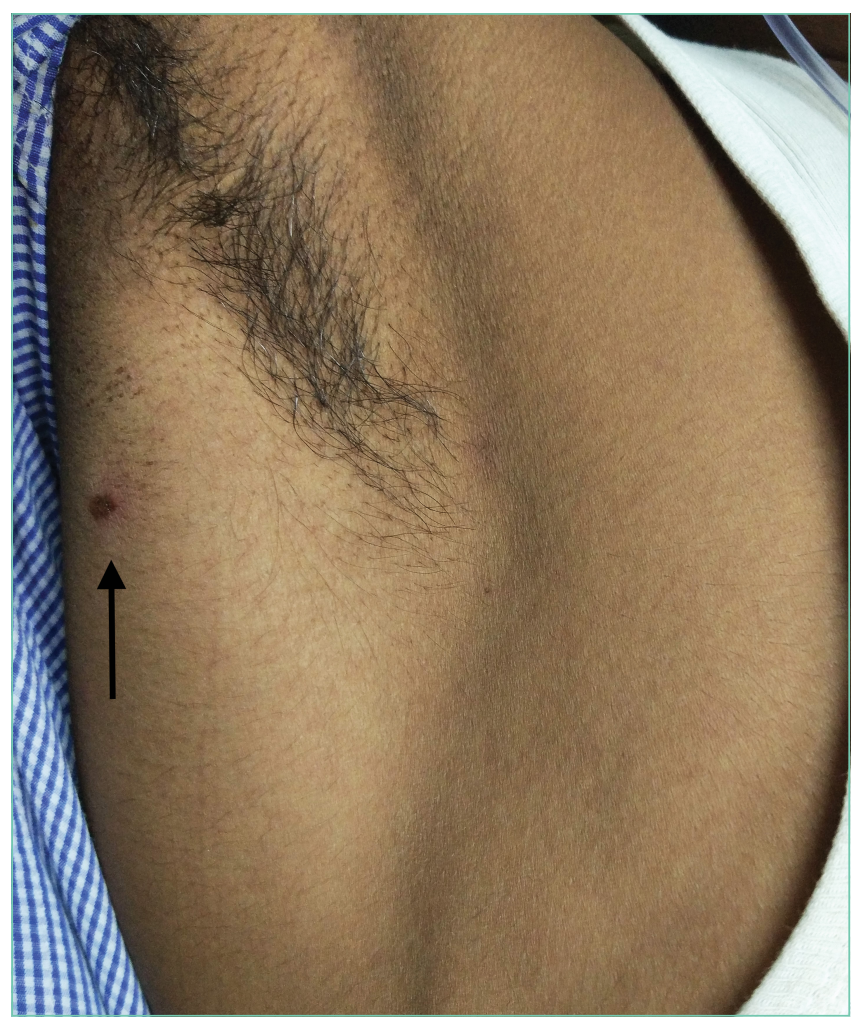

FIG 2. Eschar (arrow) in the right axillary region.

opacities and consolidation sparing the nondependent portions of the lungs. On physical inspection, a round, well-defined, painless black lesion surrounded by erythema was noticed in the right axilla (Figure 2). The rest of the examination findings were unchanged.

The discovery of eschar in the axilla provides a "pivot point" in determining the cause of the patient's illness. This finding appears to point, with high specificity, toward rickettsia as the explanation of the patient's disease, and this is most likely to be scrub typhus. The report of a positive dengue IgM may repre- 
sent concurrent infection or may simply reflect a recent infection in an area that is highly endemic for dengue. Although most of the patient's clinical presentation could be attributed to dengue, multiple features including the leukocytosis, myocarditis, and elevated bilirubin are more likely to be seen in scrub typhus. In any event, dengue cannot satisfactorily explain the eschar.

No mention has been made to the initiation of doxycycline thus far; this agent needs to be started promptly. Polymerase chain reaction (PCR) testing for scrub typhus should be ordered if available; if not, acute and convalescent serology may be obtained.

Given the finding of axillary eschar, the patient was diagnosed with scrub typhus. Doxycycline $100 \mathrm{mg}$ by nasogastric tube twice a day was initiated. The patient began to show marked symptomatic improvement. His P:F ratio improved, and he was successfully weaned off and extubated after 24 hours. Postextubation, he was kept on BiPAP for 12 hours. He was transferred out of the ICU and monitored for 72 hours. With therapy, his cytopenias, liver and renal function, and ECG normalized. Indirect immunofluorescence assay for scrub typhus returned positive at a dilution of $>1: 512$. PCR assay targeting the $56 \mathrm{kDa}$ region of Orientia tsutsugamushi was also positive. Repeated TTE showed an LVEF of $65 \%$. He was subsequently discharged with oral doxycycline and a plan to complete a course of 14 days on an outpatient basis. The final diagnosis was scrub typhus with myocarditis leading to acutely decompensated heart failure with reduced ejection fraction.

\section{DISCUSSION}

Scrub typhus is a mite-borne tropical infection caused by the gram-negative intracellular parasite Orientia tsutsugamushi from the Rickettsiaceae family that is known to occur in certain parts of Asia and Australia. Although this entity is well known in the Sub Himalayan belt and southern part of India, very few cases have been described in Delhi, the capital state in North India. Scrub typhus, like most other tropical infections, is found most often during the postmonsoon season. ${ }^{1,2}$

Patients with scrub typhus present with fever in addition to a variety of nonspecific symptoms and findings. These often manifest within 10 days of being bitten by a mite. Malaise, headache, myalgias, lymphadenopathy, and maculopapular or petechial rash are common. If present, the rash manifests on the $3^{\text {rd }}$ to $5^{\text {th }}$ day of fever. ${ }^{3}$ Disseminated vasculitis due to scrub typhus can frequently result in multiorgan system involvement. Pulmonary involvement often leads to acute respiratory distress syndrome (ARDS) with an incidence of $8 \%-10 \% .{ }^{1,4}$ Acute kidney injury, mostly mild and nonoliguric, has been reported in up to $2 / 3$ cases. ${ }^{4-6}$ The cardiac myocyte is a known target cell affected by scrub typhus, and therefore patients commonly present with myocarditis. ${ }^{7}$ Liver involvement in scrub typhus is evident through elevated liver enzymes and can occur without other clinical evidence of the illness. ${ }^{4,6,8,9}$ As in dengue, patients often develop thrombocytopenia, but normal hemoglobin in scrub typhus differentiates it from dengue..$^{6,8}$
Given the nonspecific presentation, it can be challenging to diagnose and treat scrub typhus. The gold standard for diagnosis is the detection of IgM antibodies to Orientia tsutsugamushi using an indirect immunofluorescence assay (IFA). For patients from endemic regions, it may be necessary to show a four-fold increase in titers two weeks apart to distinguish from background immunity. Presence of the characteristic eschar, as discussed below, is highly suggestive of scrub typhus. The treatment of choice is doxycycline or azithromycin for seven days. ${ }^{10,11}$ Early initiation of doxycycline when considering either scrub typhus or leptospirosis is appropriate and may be life-saving.

Medical decision making is fraught with uncertainty, and physicians must use their experience, evidence base, and cognitive heuristics wisely to care for patients effectively. For this patient, the region of Delhi experiences massive outbreaks of dengue every year during the time the patient presented to the hospital, whereas rickettsia infections are relatively uncommon. The clinical presentation was conceivably consistent with either dengue or scrub typhus, though somewhat more suggestive of the latter. Once the serological diagnosis of recent or concomitant dengue was obtained, however, scrub typhus was considered even less. The team called upon Occam's razor or the heuristic that the simplest and most unifying explanation for any given problem is the one most likely to be correct and that other, less satisfactory explanations (in this case, scrub typhus) are "shaven off." The patient was managed conservatively for dengue. Only when his condition worsened did the team recognize this conflicting information without dismissing it, consider alternative possibilities, and reexamined the patient.

An eschar can be an important clue in the diagnosis of scrub typhus, though it is not often obvious. The presence of this necrotic skin lesion with black crust is highly suggestive of scrub typhus, and in the right clinical context, it is virtually diagnostic. However, it is uncommon (9.5\%-45\%) in most of the studies from the Indian subcontinent (ie, high specificity but low sensitivity). ${ }^{1,12}$ An eschar is often found in obscure locations such as the axillae or groin, areas that may easily be missed or overlooked. Eschars may be seen in a variety of other infectious diseases, including rickettsia pox, Rocky Mountain spotted fever, other members of the spotted fever group, tularemia, and cutaneous anthrax. Given this patient's lack of improvement, repeated examination revealed an eschar in the right axilla, a finding that was either missed or still evolving at the time of presentation.

This case illustrates the challenges in interpreting the significance of multiple positive serological tests in the context of an undifferentiated clinical syndrome. Possible reasons for a positive dengue serology could have been persistent antibodies from a previous infection, recent asymptomatic infection, concurrent infection, or cross-reactivity with flaviviruses such as West Nile Virus or Japanese Encephalitis. ${ }^{13}$ The patient also had positive IgM antibodies against Legionella pneumophila, but the urinary antigen was negative. In view of a negative antigen test, low specificity of the serologic test, 
low incidence of legionellosis in the Indian subcontinent, and absence of therapeutic response to a trial of fluoroquinolones, the diagnosis of legionellosis was considered unlikely in this patient.

With rapid advancements in technology, the importance of history taking and physical examination is at risk of being overshadowed. Approximately $80 \%$ of correct diagnoses in medicine can arrive through history and physical examination alone. ${ }^{14,15}$ In this case, Occam's razor combined with multiple serological tests was relied on to create the likely list of diagnoses. However, recognition of the limitations of these heuristics and tests proved critical. The life-saving diagnosis was only made when the clinicians returned to basics, looked in every nook and cranny, and found the eschar on physical examination.

\section{KEY TEACHING POINTS}

- In patients living in endemic areas who present with an acute febrile illness, the differential diagnosis should include "tropical" infections such as dengue, chikungunya, enteric fever, leptospirosis, malaria, and scrub typhus.

- Serology is commonly employed for diagnosis of tropical infections, which may be misleading. These tests can be falsely positive from past asymptomatic infection or cross reactivity between antibodies, or falsely negative, as in the first few days of infection.

- Presence of eschar is a very useful clue in the diagnosis of scrub typhus, but this finding can be missed since it is often found in obscure locations. A thorough clinical history and physical examination are paramount.

Disclosures: The authors do not report any conflict of interest.

\section{References}

1. Gupta N, Chaudhry R, Kabra SK, et al. In search of scrub typhus: a prospective analysis of clinical and epidemiological profile of patients from a tertiary care hospital in New Delhi. Adv Infect Dis. 2015;5(4):140. doi: 10.4236/ aid.2015.54017.

2. Kamarasu K, Malathi M, Rajagopal V, Subramani K, Jagadeeshramasamy D, Mathai E. Serological evidence for the wide distribution of spotted fevers \& typhus fever in Tamil Nadu. Indian J Med Res. 2007;126(2):128-130.

3. Mahajan SK. Scrub typhus. J Assoc Physicians India. 2005;53:954-958.

4. Mahajan SK, Rolain JM, Kashyap R, et al. Scrub typhus in the Himalayas. Emerg Infect Dis. 2006;12(10):1590-1592. doi: 10.3201/eid1210.051697.

5. Attur RP, Kuppasamy S, Bairy M, et al. Acute kidney injury in scrub typhus. Clin Exp Nephrol. 2013;17(5):725-729. doi: 10.1007/s10157-012-0753-9.

6. Varghese GM, Trowbridge P, Janardhanan J, et al. Clinical profile and improving mortality trend of scrub typhus in South India. Int $J$ Infect Dis. 2014;23:39-43. doi: 10.1016/j.ijid.2014.02.009.

7. Taylor AJ, Paris DH, Newton PN. A systematic review of mortality from untreated scrub typhus (Orientia tsutsugamushi). PLoS Negl Trop Dis. 2015;9(8):e0003971. doi.org/10.1371/journal.pntd.0003971

8. Chrispal A, Boorugu H, Gopinath KG, et al. Scrub typhus: an unrecognized threat in South India-clinical profile and predictors of mortality. Trop Doct. 2010;40(3):129-133. doi: 10.1258/td.2010.090452. 9. Mathai E, Rolain JM, Verghese GM, et al. Outbreak of scrub typhus in southern India during the cooler months. Ann N Y Acad Sci. 2003;990:359-364. doi: 10.1111/j.17496632.2003.tb07391.x

10. Gupta N, Chaudhry R, Kabra SK, et al. Comparative evaluation of serological and molecular methods for the diagnosis of scrub typhus in Indian settings. Jpn J Infect Dis. 2017;70(2):221-222. doi: 10.7883/yoken.JJID.2016.139.

11. Rahi M, Gupte MD, Bhargava A, Varghese GM, Arora R. DHR-ICMR Guidelines for diagnosis \& management of Rickettsial diseases in India. Indian J Med Res. 2015;141(4):417-422. doi: 10.4103/0971-5916.159279.

12. Sharma A, Mahajan S, Gupta ML, Kanga A, Sharma V. Investigation of an outbreak of scrub typhus in the Himalayan region of India. Jpn J Infect Dis. 2005;58(4):208-210.

13. Gupta N, Chaudhry R, Mirdha B, et al. Scrub typhus and leptospirosis: the fallacy of diagnosing with IgM enzyme-linked immunosorbent assay. J Microb Biochem Technol. 2016;8:71-75. doi: 10.4172/1948-5948.1000265.

14. Peterson MC, Holbrook JH, Von Hales D, Smith NL, Staker LV. Contributions of the history, physical examination, and laboratory investigation in making medical diagnoses. West J Med. 1992;156(2):163-165. doi: 10.1097/00006254199210000-00013

15. Roshan M, Rao AP. A study on relative contributions of the history, physical examination and investigations in making a medical diagnosis. $J$ Assoc Physicians India. 2000;48(8):771-775. 